\title{
L'intertextualité dansRue des voleurs de Mathias Énard
}

\author{
Nader Anwar Hénawi Boutros
}

Maître de Conférences Département de français

Faculté Al Alsun Université d'Ain Chams

\section{Résumé}

Dans cette recherche, nous parlons de l'Intertextualité dans "Rue des voleurs" de Mathias Énard. Nous évoquons le lien implicite qui existe entre l'histoire principale du héros et les intertextes qui apparaissent dans le roman. Dans ce livre, nous avons égard à Lakhdar, un jeune musulman de Tanger, qui abandonne la maison de son père après avoir été surpris en train de faire l'amour avec sa cousine. Depuis ce jour, la vie du jeune homme se transforme en une série de calamités. Notre problématique consiste alors à étudier comment les histoires secondaires, insérées dans $\underline{\text { Rue des voleurs, }}$ représentent la nature de l'Homme qui se voit tiraillé entre deux postulations contradictoires: le Bien et le Mal.

Tout le long de cette recherche, nous recourons à une approche comparatiste puisque nous établissons des corrélations entre le récit d'apprentissage de Lakhdar, et l'intertexte, d'autre part. Tout d'abord, nous parlons d'un mythe très répandu dans toutes les croyances et les religions monothéistes: celui du péché originel. Nous soulignons les invariants et les variantes qui existent entre le récit de Lakhdar et le mythe du paradis perdu. À travers l'énumération des malheurs de Lakhdar en dehors du foyer parental, nous nous assurons que cette maison représente le paradis perdu, où l'on jouit gratuitement de tous les plaisirs. Nous abordons aussi l'hybridité du texte d'Énard qui accorde une place considérable à la langue arabe et au métissage culturel.

Dans la conclusion de la recherche, nous mettons l'accent sur l'originialité de Mathias Énard qui ne se contente pas de rassembler les textes antérieurs, mais qui les dote 
également d'une valeur supplémentaire à travers l'aventure de son héros.

Les mots-clés: l'approche comparative- le bilinguismel'hybridité du texte- l'hypotexte-l'hypertexte-l'imitation.

\section{Introduction.}

Écrivain français, Mathias Énard étudie l'arabe et le persan. Il va même jusqu'à enseigner l'arabe à l'université autonome de Barcelone. En 2012, il publie Rue des voleurs où il relate le récit de Lakhdar, un jeune Marocain de Tanger, un jeune musulman passable, juste trop avide de liberté et d'épanouissement, dans une société où la moindre faute morale est sévèrement sanctionnée. Suite à un adultère commis avec Meryem, sa cousine, Lakhdar est chassé de la maison parentale. Il devient alors sans domicile ni profession. Commence ensuite un dur périple où il ne connaît que besoin et humiliation.

Si nous avons choisi ce roman comme objet d'étude, c'est parce qu'il rassemble des intertextes faisant écho à l'aventure du héros, comme si le périple ou le trajet initiatique de Lakhdar se reconstituait non à travers l'intrigue ou les actions du personnage, mais à travers les fragments littéraires et culturels éparpillés çà et là dans Rue des voleurs.

Dans le domaine littéraire, le texte ne se crée jamais « ex nihilo ». $\mathrm{Au}$ contraire, il y a toujours des " similitudes aléatoires »(WACH, 2012: 365) entre les divers textes. En fait, l'empreinte des autres écrivains doit toujours apparaitre dans tout livre car l'ouvrage littéraire dialogue toujours avec les textes présents, passés et à venir, comme en témoigne Julia Kristeva:

"Tout texte se construit comme une mosaïque de citations, tout texte est absorption et transformation d'un autre texte. »(KRISTEVA, 1969: 85)

Pour ce qui est de Rue des voleurs, Énard a dû emprunter quelques idées aux écrivains antérieurs. Ces idées ne s'avèrent 
donc pas tout à fait neuves car tout texte comprend en lui d'autres textes, mais à différents degrés. Ce phénomène est connu dans la littérature sous le nom d'"Intertextualité". Les lignes ci-dessous servent à définir ce terme selon le point de vue de Riffaterre:

"L'intertextualité est la perception par le lecteur, de rapports entre une ouvre et d'autres, qui l'ont précédée ou suivie. "(RIFATERRE, 1980: 4)

De façon brève, l'intertextualité souligne qu'il y a un lien de dépendance entre un texte initial ou un mythe d'une part, et l'autre texte, celui que nous choisissons comme objet d'étude, d'autre part.

Par ailleurs, les spécialistes dans le domaine de la littérature comparée définissent 1"'Intertextualité" comme étant

"le travail de transformation et d'assimilation de plusieurs textes opéré

par un texte centreur qui garde le leadership du sens. " (CLAUDON \& al., 1992: 22)

Selon Gérard Genette, le texte initial ou le mythe s'appelle "hypotexte" alors que le texte récent s'appelle "hypertexte" (GENETTE, 1982: 110). D'après ce théoricien, l'hypertexte peut conserver l'hypotexte, le supprimer (problèmes de la trace), le modifier ou le transformer (ancien problème des sources) ou bien le développer (glose, amplification).

Dans la présente recherche, nous chercherons à étudier comment les intertextes insérés au sein du texte d'Énard dévoilent l'essence des êtres ainsi que la nature de l'univers où nous vivons. Plutôt que de mettre l'accent sur les déambulations de Lakhdar à travers l'espace et le temps du roman au sens linéaire, nous mettrons en relief ses déplacements dans "un espace-temps révolu » (BONI, 2011: 124)., c'est-à-dire dans des mondes hybrides et des cultures archaïques. En d'autres termes, notre étude tend à focaliser sur 
les intertextes et les récits secondaires, perçus toujours comme des digressions narratives ou de simples fioritures reléguées à l'arrière-plan.

Quant à la méthode que nous emploierons, nous appliquerons l'approche « comparatiste » puisque nous établirons des corrélations entre l'histoire principale de Lakhdar et les intertextes. Nous soulignerons les invariants (les points de convergence) et les variantes (les points de divergence) entre le récit d'apprentissage du héros, d'une part et les allusions faites aux histoires secondaires, d'autre part.

En abordant l'Intertextualité, nous nous fonderons sur les notions publiées par Danie-Henri Pageaux dans son livre intitulé "La Littérature générale et comparée » (1994) ainsi que sur les théories publiées par Didier Souiller dans son essai intitulé "Littérature comparée »(1997). Tous les deux affirment que l'écrivain insère inconsciemment des textes déjà lus dans les ouvrages antérieurs. Selon ces théoriciens, l'intertextualité ne signifie pas qu'il y a une "imitationdécalque" des oeuvres antérieures, mais qu'il y a une imitation créatrice du passé. Même s'il soumet son écriture à un inconscient collectif et même si l'oeuvre abonde en renvois, l'auteur doit faire preuve d'originalité. Parviendrons-nous à dévoiler les aspects de cette originalité chez Mathias Énard? C'est ce que nous essaierons de voir à travers les pages suivantes.

\section{Le mythe du paradis perdu.}

Dès l'incipit de Rue des voleurs, Mathias Énard fait appel à la "mémoire culturelle" (WACH, 2012: 23) du public. En fait, il fait renaître les mythes du péché originel et du paradis perdu puisque le lecteur peut bien trouver une empreinte biblique ou une allusion à la Genèse, dans laquelle Adam, tenté par le diable à travers Ève, accepte une pomme et est, de ce fait, chassé du Paradis, sous la fureur de Dieu. Dans le roman 
d'Énard, nous avons égard au même épisode de la tentation qui exerce une

" influence inconsciente » (WACH, 2012: 365) sur l'auteur puisque nous avons égard à un héros qui ne peut pas résister au charme de Meryem, sa cousine. Il fait l'amour avec elle et décrit cet acte comme étant un "péché originel " (ÉNARD, 2012: 89).

En effet, Meryem prend l'image du démon tentateur:

«(...) à la maison elle portait souvent des jeans moulants ou des robes d'intérieur à demi transparentes, (...) elle m'excitait terriblement, (...) et dans mes rêveries érotiques avant de m'endormir je m'imaginais la déshabiller, (...), mais j'aurais été incapable de faire le premier pas. " (ÉNARD, 2012: 12)

Meryem passe donc pour une femme fatale qui séduit Lakhdar, son cousin. Celui-ci est tellement obsédé par ses attraits sexuels qu'il en rêve toujours et qu'il s'avère incapable de refouler ses désirs. En effet, cette jeune fille occupe une place considérable dans la vie inconsciente du héros qui couche avec elle après avoir reçu le premier feu vert:

" (...) je l'avais déshabillée presque sans vouloir, timidement, après qu'elle m'eut embrassé sur la bouche en me tenant la main (...). » (ÉNARD, 2012: 34)

Meryem est donc la source du Mal. C'est elle qui séduit son cousin et qui l'entraîne au péché. À cet égard, Lakhdar fait allusion à «Joseph que tente Zuleykha »(ÉNARD, 2012: 34).

L'histoire de Joseph nous montre ici que Lakhdar est tiraillé entre deux postulations, entre deux voix. L'une est celle de la vertu qui exige que l'homme déploie un effort pour lutter contre les mauvais penchants. Quant à l'autre voix, c'est celle de l'épicurisme et du vice qui invite l'être à tirer profit de tous les plaisirs terrestres, abstraction faite de la morale et de la religion. Ainsi l'épisode de la tentation de Joseph montre-t-il à Lakhdar qu'il aurait dû lutter contre le vice car 
"à chaque génération, le mythe rappelle qu'il est donné à l'homme de choisir sa vie, d'être créateur ou négateur de soi. » (PAGEAUX, 1994: 109)

Si Joseph a été emprisonné et s'il a été enfermé dans un espace clos, Lakhdar a été chassé de la maison familiale, donc il a connu le besoin, le

danger et l'humiliation. Toutefois, la souffrance de Joseph était momentanée puisque l'emprisonnement était un motif de sa promotion ultérieure et de son ascension sociale et morale dans la cour égyptienne. Pour ce qui est du malheur de Lakhdar, il est sans fin: il sort d'une mésaventure pour être entraîné dans une autre. Jusqu'à la fin du roman, il ne fera qu'errer et s'enliser dans la médiocrité, comme si la résistance aux désirs sordides était l'unique moyen qui assure à l'être non seulement le salut ici-bas mais aussi dans l'au-delà, alors que le fait de succomber à la tentation ne vaut à l'être que misère et opprobre.

Cette condition lamentable est due au fait que le Cheikh Mohsen, le père de Lakhdar, prend l'image de Dieu qui a chassé Adam du paradis terrestre. Après avoir surpris son fils à poil avec Meryem, le père riposte de façon catégorique et intransigeante:

"Quelques mois plus tard je prenais ma première trempe, une avalanche de beignes comme je n'en avais jamais connu, (...), mon père

pleurait lui aussi, de honte, et il récitait des formules de conjuration, Dieu nous protège du malheur, Dieu nous aide, (...) ") (ÉNARD, 2012: 14)

À la différence de l'histoire d'Adam et Ève punis par Dieu, Lakhdar et Meryem sont punis par le Cheikh Mohsen (donc un être humain ordinaire) qui détient la raison et la loi et qui symbolise le pouvoir absolu. C'est ainsi que ce père incarne les valeurs du surmoi et qu'il exerce "une justice vengeresse, répressive et sévère »(SOUILLER \& al., 1997: 621). Tous les deux, Lakhdar et sa cousine, doivent payer le prix de leur 
erreur. En ce qui concerne Meryem, elle a été envoyée vivre chez sa tante au fin fond du Rif. Enceinte, elle a écrit des lettres qui n'avaient été envoyées à Lakhdar qu'après la mort de celle-là. Dans ces lettres, elle sollicitait l'aide de Lakhdar qui devrait l'épouser, sinon elle devrait abandonner l'enfant à l'orphelinat. À la différence d'Ève qui a longtemps vécu suite à son expulsion du paradis terrestre, Meryem est morte tout de suite. Si Ève enfante dans la douleur, Meryem ne donne pas naissance à son enfant à cause de "son hémorragie au cours d'un avortement paysan et clandestin »(ÉNARD, 2012: 79).

Pour ce qui est de Lakhdar, lui, il doit accomplir le même trajet effectué par Adam. Il doit gagner son pain à la sueur de son front car son père le déshérite. N'ayant que dix dirhams en poche, il ne sait où aller ni où dormir. Il est chassé à jamais de la maison parentale, voire de son paradis terrestre:

«(...) je savais que je ne rentrerais pas chez moi, que je ne rentrerais plus, c'était impossible. »(ÉNARD, 2012: 15)

En général, le texte de Rue des voleurs est rythmé par le sentiment de la

nostalgie qu'éprouve le héros à l'égard du foyer paternel. Loin de ce paradis perdu qui représente "le repos domestique, familial, maternant »(KRISTEVA, 1977: 210), Lakhdar doit connaître le vieillissement précoce, comme si les habitants de la maison parentale jouissaient de la jeunesse durable:

" je détaille les fils de cheveux blancs sur mes tempes, mes yeux noirs, mes mains aux ongles rongés; je m'interroge sur ma culpabilité.» (ÉNARD, 2012: 251)

En dehors de ce foyer, Lakhdar doit faire face à la solitude et ne doit rien attendre des autres; si le père biologique refusait de lui pardonner ou de renouer avec lui, les étrangers l'aideraient-ils? Ainsi le héros de Rue des voleurs doit-il faire face à toutes les épreuves: peur de l'inconnu, errance, froid, violence, avances des ivres et des homosexuels qui tentent de 
le violer en public. Par contre, pendant qu'il était au foyer, il jouissait de tous les éléments dont il avait besoin pour s'épanouir: sécurité, nourriture, refuge et havre de paix. À l'intérieur de la maison parentale, on ne mangeait que des fruits frais, alors que les «fruits pourris » se trouvent hors de ce paradis:

" J'ai eu faim, j'ai bouffé des fruits pourris que les marầchers laissaient aux mendiants, j'ai dîu me battre pour des pommes mâchées, puis des oranges moisies, (...); j'ai eu froid, j'ai passé des nuits trempé à l'automne, quand les orages s'abattaient sur la ville (...). » (ÉNARD, 2012: 17)

À l'instar d'Adam qui a été condamné à déployer un effort pour gagner son pain, le héros de Rue des voleurs doit travailler pour survivre. Tout d'abord, il travaille dans une librairie dans le Groupe musulman pour la Diffusion de la Pensée coranique. Or cette librairie est incendiée. Quelques mois après, il travaille comme dactylo chez M. Jean-François chez qui le travail est " pénible, aride et abrutissant » (ÉNARD, 2012: 95).

Il travaille également sur un ferry-boat où les conditions de travail s'avèrent plus cruelles:

"Mon salaire tardait à arriver, on ne me payait pas, mes économies se réduisaient à pas grand-chose; le boulot était assez fatigant et monotone.» (ÉNARD, 2012: 129)

Instabilité et insécurité sont donc le lot réservé à ce garçon qui a

commis un péché et qui, par ce fait, a perdu le paradis représenté par le foyer. Il doit aussi travailler avec M. Cruz dans une morgue où il s'occupe des morts. La précarité de sa condition l'oblige même à s'occuper du ménage ainsi que de l'entretien du jardin de M.Cruz, à laver sa voiture et à nourrir ses chiens.

Même lors de son séjour en Espagne, la situation économique de ce 
pays allait de mal en pis, à cause de la grève des travailleurs et de la révolte menée par le mouvement des Indignés. Il lui est donc difficile de trouver un emploi en Europe ou même d'y continuer ses études universitaires. S'il était un maître dans sa maison parentale, le péché a fait de lui un esclave.

Dans le passage ci-dessous, le héros de Rue des voleurs finit par faire le bilan de son expérience:

"la vraie vie n'avait toujours pas commencé, sans cesse remise à plus tard: ajournée à la Diffusion de la Pensée coranique partie en flammes; différée sur l'Ibn Batouta, embarcation perdue; retardée chez Cruz, chien parmi les chiens; suspendue à Barcelone au bon vouloir de la crise (...). » (ÉNARD, 2012: 218)

Aucune promesse de bonheur ne se profile donc à l'horizon. Nous avons plutôt égard à un être désarmé face au destin. Le bonheur absolu auquel il aspire est un leurre, un mirage difficile à atteindre. Nous avons donc égard à «une écriture de la perdition, de l'égarement, en somme du labyrinthe retrouvé »(DETHURENS \& al., 2000: 105).

En continuant la lecture de Rue des voleurs, nous nous assurerons que Mathias Énard a fait de son roman un creuset de plusieurs cultures, un texte hétérogène et hybride par excellence.

\section{L'hybridité du texte.}

Tout d'abord, Lakhdar fait allusion au célèbre roman autobiographique

intitulé Le Pain nu de l'écrivain marocain Mohamed Choukri. C'est l'un des premiers textes qui aborde des sujets tabous dans la société nord-africaine de l'époque, comme la drogue, la violence ou la sexualité. En lisant ce livre, le narrateur-héros ne peut pas cacher son admiration à l'égard de Choukri:

"Entre-temps, j'avais lu Le Pain nu,(...) : ce Choukri était hors du commun. Son arabe était sec comme les coups de 
trique qu'il recevait de son père, dur comme la famine. " (ÉNARD, 2012: 108)

En effet, Le Pain nu raconte l'enfance et l'adolescence de Mohamed Choukri qui a suivi sa famille dans son exode depuis le Rif jusqu'à Tanger. Le texte reprend la figure du père, figure haïe du narrateur, d'un père alcoolique, violent, et même meurtrier. Mohamed sombre peu à peu dans l'alcool et la drogue. Il connaît la vie des rues et décrit la violence qu'il vit au jour le jour. Il fréquente le milieu de la prostitution. En lisant tous ces détails, Lakhdar s'identifie à Choukri:

"Il n'avait pas peur, il racontait sans rien dissimuler, ni le sexe, ni la violence, ni la misère. Ses errances me rappelaient mes mois de vagabondage. » (ÉNARD, 2012: 108)

Tous les deux, Lakhdar et Choukri, errent à Tanger et subissent les coups de leurs pères. Cependant, le père de Lakhdar n'est pas alcoolique. Au contraire, il est pieux et intransigeant en matière de religion. Cette situation montre que la modération est une vertu nécessaire. En effet, sévérité et extrémisme religieux ont des conséquences néfastes et engendrent une frustration considérable chez les générations à venir, chez ces jeunes qui vivent avec des pères à la mentalité étriquée. D'ailleurs, Lakhdar diffère de Choukri qui est «un pauvre type, un mendiant analphabète, un dégénéré » (ÉNARD, 2012: 87). En effet, le héros de Rue des voleurs a déjà reçu une certaine éducation et a appris l'arabe, le français et l'espagnol. Il a tellement confiance dans l'avenir qu'il ne s'enlise jamais dans le pessimisme absolu, comme en témoigne la citation ci-dessous:

"On trouve toujours plus misérable que soi, (...) j'avais un peu d'éducation, un peu d'argent et un pays où, dans le pire des cas, on pouvait vivoter - j'étais un enfant de la ville, j'avais lu des livres, je parlais des langues étrangères, je savais me 
servir d'un ordinateur, je finirais bien par trouver quelque chose » (ÉNARD, 2012: 149)

Toutefois, le héros de Rue des voleurs adopte la même attitude que celui du Pain nu. Si Choukri consomme de la drogue pour oublier ses malheurs, Lakhdar fume un bout de Kif. Il recourt donc à la même solution, simple mais grossière, à la solution néfaste qui empire la situation et qui accentue sa crise au lieu de l'éradiquer.

Il en est de même pour la poésie qui passe aussi pour une solution, voire une échappatoire aux malheurs de Lakhdar. Celui-ci cherche à écrire des poèmes pour ses deux bienaimées: Meryem et Judit (la jeune Espagnole qui vient étudier à Tanger). Mais en se lançant dans ce projet, il reçoit un choc affreux car il reconnaît son échec et son impuissance à écrire des poèmes de bonne qualité.

Le jeune homme se voit ainsi tiraillé entre deux tendances contradictoires: création et imitation, souci de compter sur soi et désir de compter sur les autres, indépendance et servilité. Toutefois, il finit par emprunter les vers à Nizar Kabbani:

«Aveuglé, je ne pensais plus qu'à l'arrivée de Judit, à ces vers trop sentimentaux de Nizar Kabbani que nous recopiions, au lycée, dans des messages secrets pour des filles qu'ils émouvaient, ceux que j'avais déjà récités à Meryem, عيناك أخر "Tes yeux sont comme deux derniers bateaux en partance, pourrais-je y trouver place?" alors que nous contemplions le Détroit, sans oser nous prendre par la main, (...) »

Si Lakhdar emprunte des poèmes à Kabbani, c'est parce qu'il s'avère incapable d'égaler ou de surpasser les poètes arabes. Outre l'hybridité du texte d'Énard qui résulte du mélange des genres et des styles, il y a ce métissage linguistique qui provient des vers écrits en arabe au sein d'un roman français. En effet, nous avons égard à un écrivain cosmopolite qui puise dans le trésor de la langue arabe, qui 
réhabilite ce langage et qui s'oppose ainsi à certains écrivains malhonnêtes et mercenaires considérant l'arabe comme une " langue morte »(KROH, 2000:129).

En insérant l'arabe dans son roman, Énard veut que le lecteur français soit avisé du charme de cette langue orientale qui accorde une place considérable à la poésie courtoise et à l'amour raffiné. Si l'amour physique obsède Lakhdar, celuici est aussi attiré par l'art poétique et sublime qui place la femme au-dessus de tout le monde.

Que ce soit dans le cas de Lakhdar ou de Kabbani, nous avons égard à un héros souffrant d'une crise psychologique. Seule la présence de la femme pourrait soulager cet homme de ses angoisses et peines. En répétant ces vers et même en les traduisant vers le français, Lakhdar confirme son tiraillement entre deux mondes opposés: corps et esprit, sentiments charnels et sentiments éthérés. C'est ainsi qu'il cherche à communiquer au lecteur français qu'au-delà de la nature lubrique de l'homme oriental, il y a une tendance spirituelle qui pousse ce dernier à rechercher une femme idéale apte à le comprendre et à le contenir.

Si Lakhdar lit Nizar Kabbani et s'il imite son écriture, il lit aussi des écrivains espagnols qui lui permettent de savoir comment les Occidentaux perçoivent Tanger, la ville natale du héros:

"Il y avait une section de littérature tangéroise, et ils étaient tous là, les auteurs que Judit avait évoqués: Bowles, Burroughs, Choukri bien sûr, mais aussi un Espagnol du nom d'Ángel Vázquez, qui avait écrit un roman appelé La Chienne de vie de Juanita Narboni- ce que je cherchais dans les livres c'était plutôt oublier ma chienne à moi, oublier Tanger (...). » (ÉNARD, 2012: 81-82)

Dans ce roman, Juanita, l'héroïne qui vit à Tanger, raconte son histoire dans une sorte de monologue tout en faisant de Tanger une ville si contradictoire. 
En effet, le Tanger de Rue des voleurs ressemble beaucoup à celui

du livre espagnol. Dans le roman d'Énard, cette ville se caractérise par des oppositions flagrantes, comme en témoigne l'exemple ci-dessous:

" il y en a qui espèrent se taper des princesses des Mille et Une Nuits, (...) tant ils associent à Tanger une sensualité, un désir, une permissivité qu'elle n'a jamais eue pour nous, mais qu'on offre au touriste moyennant espèces sonnantes et trébuchantes dans l'escarcelle de la misère. " (ÉNARD, 2012: 11-12)

C'est ainsi que Tanger revêt deux images contrastées. L'une, qui est apparente, est liée à la sensualité, image inculquée par Mille et Une Nuits, ce livre qui fait de la femme orientale une " femme objet" et qui souligne son charme exotique ainsi que sa totale soumission à l'homme. En ce qui concerne l'autre image, qui est plus réelle, c'est celle de la frustration destinée à emprisonner le héros dans les prières et à le priver de tous les plaisirs. La société tangéroise représente alors le surmoi qui réprime les instincts des jeunes, lesquels placent le sexe parmi leurs priorités et se complaisent à convoiter les touristes, incarnant ainsi une avidité sexuelle qui dépasse les limites. C'est ainsi que Lakhdar assume le rêve de liberté de la libido dans une société répressive en matière sexuelle, dans une société " castratrice-légiférante » (KRISTEVA, 1977: 207). Il devient incapable de jouir de son existence ou de goûter les délices du monde à cause du milieu tangérois qui cherche "à imposer un ordre, à contenir (...) les lois de la concupiscense et l'impétuosité des désirs masculins. » FAUCONNIER, 2006: 48)

Il paraît ainsi que Tanger incarne une ambivalence flagrante car si nous approfondissons notre étude de Rue des voleurs, nous trouverons que le café tangérois "Chez Mehdi" est un espace dont on ne peut pas déterminer les orientations et 
qui mêle toutes les traditions et les identités. En parlant avec Judit de Tanger, Lakhdar a l'impression

"d'évoquer une ville différente, deux images, deux territoires étrangers reliés par un même nom, une erreur d'homophonie » (ÉNARD, 2012: 63).

Il y a toujours cette dualité qui caractérise les personnages et les lieux. Nous vivons à une époque où se multiplient les croyances, les attitudes et les points de vue. Chaque être revêt un double aspect, une double image. Nous avons toujours égard à " une dialectique des contraires » (MAURON, 1996: 63). Par conséquent, il est difficile de préciser la véritable nature de Tanger qui passe pour un mystère insondable. Ainsi Lakhdar se sent-il dépourvu de toute identité. Égaré, il devient incapable de trancher la situation et de préciser ce qu'il veut: tantôt il pense à "Zahra" (une prostituée qui a des tendances exhibitionnistes), à ses comportements et à ses attitudes provocatrices, tantôt il repousse les initiatives de cette femme et refuse de coucher avec elle.

Même en faisant l'amour avec Judit, Lakhdar est aussi pris de honte et de culpabilité. Si Tanger a un double aspect, Lakhdar (le tangérois) se voit, à son tour, tiraillé entre deux postulations contradictoires: le péché et la morale. Tantôt il souligne son caractère réservé et timide, tantôt il passe pour un être lubrique qui donne libre cours à ses pulsions et qui adopte la philosophie épicurienne dont parle Michel Monteaux dans ses écrits:

"Maintenant, on ne se sent plus coupable quand on a des plaisirs illicites, comme avant, mais quand on n'est pas capable d'en profiter, quand on ne parvient pas à jouir. » (MONTEAUX, 2006: 32)

Parmi les aspects qui font de Tanger une ville à double identité, il y a la montée des Islamistes au pouvoir et l'ascension des Frères Musulmans qui s'opposent à la laïcité du 
pays. Ce courant est symbolisé dans Rue des voleurs par le Cheikh Nouredine qui, par ses idées et actes, incarne un courant extrémiste. En guettant cet homme, Lakhdar se formule des opinions personnelles relevant de son apprentissage. Grâce à son contact avec Nouredine, Lakhdar devient conscient de la notion du Djihad.

\section{Le Coran et la notion du Djihad.}

Tout d'abord, Nouredine (l'homme qui accueille Lakhdar à la suite de

son expulsion du foyer parental) s'insurge farouchement contre l'Islam ascétique et spirituel. Il défend l'Islam politique et refuse de séparer la religion des questions publiques. Les quelques lignes ci-dessous jettent la lumière sur un sermon prononcé par Nouredine:

«Ils (Les auditeurs) sortaient par bandes d'une dizaine, armés de gourdins et de manches de pioches après un sermon belliqueux et éloquent du Cheikh Nouredine, où il était question des expéditions du prophète, du combat de Badr, du Fossé, (...) de la gloire des martyrs en paradis. " (ÉNARD, 2012: 29)

Ainsi ce Cheikh se complaît - il à rappeler aux fidèles les récits du Coran qui mettent en relief le rôle de la guerre susceptible de promouvoir la religion d'Allah. En se fondant sur des textes sacrés, Nouredine gagne la confiance et l'appui de ses camarades. Grâce à son immense capacité de raisonnement, il parvient à convaincre son groupe des bienfaits de la mort dans la bataille. Ses discours ont un effet tellement magique que ses disciples portent les armes et déclarent leur disposition à faire la guerre après avoir écouté son sermon religieux.

Cet homme, qui interprète la religion en fonction de ses propres intérêts, obsède bien Lakhdar de sorte que celui-ci se rappelle toujours ses mots qui encouragent l'attitude belliqueuse chez les musulmans: 
"(...), je les entends encore parfois dans mon sommeil, ces discours sur la bataille de Badr, Je vous viendrai en aide, avec mille anges se suivant les uns les autres, إذ تستغيثون ربك وفاستجاب لكم أني مدكم بألف من الملائكة مردفين

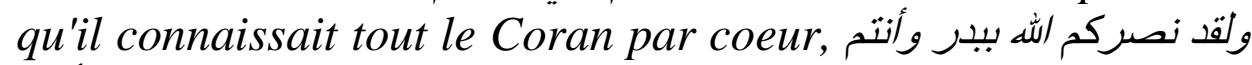
أذلة Dieu vous a donné la victoire à Badr alors que vous étiez. les plus faibles, (...)» (ÉNARD, 2012: 219)

Cet extrait tiré de la sourate d'Al-Imran jette la lumière sur la condition

des musulmans qui ont été renforcés par Dieu à Badr et qui ont triomphé de leurs ennemis. En écrivant les versets coraniques en arabe, Énard semble nous dévoiler implicitement la mentalité de Nouredine ou des Islamistes (en général) qui voient dans l'arabe une langue divine, celle du Coran.

Or il faut remarquer ici qu'en répétant ces versets, Nouredine omet un fait très important: que le passé du Prophète diffère du présent actuel. Selon Jauss, le lecteur d'un texte remontant au passé doit prendre en considération le saut temporel qui sépare le moment actuel où il parle du moment antérieur, sinon il tombera dans le piège de la subjectivité:

"Celui qui croit pouvoir atteindre l'autre horizon, celui du passé, en faisant abstraction de son propre horizon apporte immanquablement dans une reconstruction prétendument objective du passé des critères subjectifs de choix, de mise en perspective, d'appréciation. » (JAUSS, 1988: 26)

En effet, il y a un écart qui sépare l'époque du Prophète de celle des printemps arabes. Dans le VIIe siècle, il fallait qu'on lance la guerre contre les polythéistes quarychistes qui menaçaient l'Islam. Or au XXI e siècle, ces polythéistes n'existent plus et la guerre des Frères Musulmans est lancée contre l'Occident chrétien et les Juifs, et même contre les courants islamiques modérés.

En d'autres termes, Nouredine insiste toujours sur les passages coraniques qui encouragent le Djihad et oublie les 
versets qui véhiculent une attitude pacifique. Il s'accorde le droit de juger les fidèles et de les considérer comme des noncroyants puisqu'ils ont une mentalité différente de la sienne. Toute personne qui contredit sa parole, doit être supprimée, châtiée et évincée.

Tous les deux, Nouredine et Bassam (un de ses disciples), vont même jusqu'à accomplir une expédition punitive où ils humilient, injurient et battent un libraire du quartier parce qu'il vend des revues espagnoles et françaises de filles à poil. En outre, ils sont soupçonnés d'être à l'origine de l'attentat au Café Argan à Marrakech et au Café Hafa à Tanger. Nouredine aspire également à ce que les Frères Musulmans sortent grands vainqueurs de toutes les élections.

Il s'agit donc d'une attitude extrémiste et fanatique qui est commentée par Lakhdar, lequel fait preuve d'un Islam modéré:

"J'ai pensé à Bassam, perdu quelque part dans son Djihad personnel, qui avait peut-être assassiné un étudiant à coups de sabre à Tanger, (...); il n'y a rien à comprendre dans la violence, (...) dans la bêtise aveugle qui pousse un type de mon âge à poser froidement le canon d'un flingue sur la tempe d'une fillette de huit ans dans une école. " (ÉNARD, 2012: 197)

À chaque fois que Bassam accomplit un acte terroriste, Lakhdar désapprouve donc son comportement versatile et dogmatique. Celui-là s'accorde le droit de torturer, de tuer et de massacrer pour défendre la cause de Dieu. Aux yeux de Lakhdar, le Djihad de Nouredine et de Bassam n'est qu' une "rhétorique absurde »(ÉNARD, 2012: 241). Leur guerre est déclenchée " tout simplement pour le plaisir de la destruction et des flammes »(ÉNARD, 2012: 241). Ce Cheikh utilise toujours le Coran pour justifier ses attitudes belliqueuses ou hostiles envers l'Occident, comme en témoigne l'extrait cidessous où l'on trouve que Bassam récite des versets coraniques encourageant la guerre en faveur de la religion: 
"Un jour, la sourate de la Victoire: Quand viendra la victoire de Dieu et la Conquête, etc. ; un autre la sourate du Butin: Et ton Seigneur révéla aux anges: "Je suis avec vous: affermissez les croyants. Je vais jeter l'effroi dans les coeurs des impies. Frappez donc au-dessus des nuques." " (ÉNARD, 2012: 130)

لعل الساعة " Vers la fin du roman, un autre verset coranique "نكون قرييا "il se peut que l'Heure soit proche" (La Traduction $d u$ Noble Coran en langue française, 1424 de l'hégire: Sourate d'Al Ahzâb "Les Alliés", Partie 26: 427) suscite l'intérêt de Lakhdar et l'invite à commenter l'attitude de Nouredine ainsi que les actes de Bassam. Répété six fois dans le roman (ÉNARD, 2012: 240, 241, 242, 243 \& 244), ce verset "il se peut que l'Heure soit proche" devient un message implicite pour que Bassam se lance dans son Djihad et ses actes sanguinaires. Il peut maintenant mourir en faveur d'une cause sacrée: celle de la défense de la religion contre les noncroyants. Comme la mort viendra tôt ou tard et que le monde sera anéanti, à quoi servira donc le fait de s'intéresser à sa vie ici-bas?

Ainsi Nouredine insiste - $\mathrm{t}$ - il toujours, dans ses sermons, sur les histoires de "houris toujours vierges qu'on pouvait baiser pour l'éternité au bord du Kowthar, le lac d'abondance de l'au-delà »(ÉNARD, 2012: 38). En effet, cet homme se complaît à interpréter les versets coraniques de façon littérale et fait fi de l'opinion des savants mystiques qui jettent la lumière sur la valeur implicite ou la connotation symbolique des textes sacrés.

Cependant, à travers Rue des voleurs, Mathias Énard prouve que le faux Djihad, représenté par Nouredine et son groupe, a des conséquences néfastes. Tout d'abord, ce Djihad cause l'incendie du Groupe musulman pour la Diffusion de la Pensée coranique; on soupçonne le libraire tabassé par Nouredine d'être à l'origine de cet accident. Par ailleurs, le roman fait 
allusion à la fin tragique d'Oussama ben Laden. À la fin du roman, Bassam se suicide après avoir lancé un attentat terroriste.

Si le faux Djihad a de graves répercussions, le vrai Djihad assure à l'être le salut éternel. Tout le long de Rue des voleurs, Mathias Énard met en relief la notion du véritable Djihad qui réside dans la prière:

"En récitant la Fatiha, en exhalant les versets sans qu'aucune pensée ne les trouble, en répétant les mots saints, j'ai retrouvé le calme. » (ÉNARD, 2012: 92)

En fait, la Fatiha est une sourate qui dispose d'une valeur particulière dans le Coran parce qu'elle s'ouvre sur un verset mettant en relief deux attributs de Dieu "Au nom d'Allah, le Clément, le Miséricordieux" et qu'elle sollicite la soumission du croyant à la volonté divine, d'autant plus qu'elle est la seule sourate où l'on doit dire "Amine". Lakhdar semble donc être fasciné par l'Islam qui fait prévaloir la valeur du recueillement et de la prière, deux moyens grâce auxquels l'Homme peut trouver un refuge auprès de Dieu. La sourate "Al Fatiha" permet donc au croyant d'être protégé, préservé et guidé. D'ailleurs, le héros de Rue des voleurs ne trouve son bonheur qu'en s'éloignant de ces discours belliqueux prononcés par Nouredine et en contemplant les sens du Coran:

"Je passais des heures à découvrir les beautés du Coran à travers les grands commentateurs; (...). J'aimais imaginer le Prophète dans sa grotte, enveloppé dans son manteau, ou entouré de ses compagnons, en route pour la bataille. Penser que je reproduisais leurs gestes, répétais les phrases qu'ils avaient eux-mêmes psalmodiées m'aidait à supporter la prière (...). » (ÉNARD, 2012: 25)

C'est ainsi que Lakhdar éprouve une fascination à l'égard du patrimoine islamique, riche et complexe. Il s'agit, en effet, d'un nouveau courant qui fait fi de la politique et qui insiste sur le rôle de la prière et de la méditation. Plutôt que de focaliser sur 
le sang répandu et sur les stratégies adoptées par le Prophète au cours de la bataille, Lakhdar se voit attiré par le climat de convivialité entre le Prophète et ses compagnons ainsi que par la chaleur humaine qui caractérise leurs rapports. L'être humain a donc besoin d'un climat de sécurité, d'entente et de paix. C'est ainsi que le véritable Djihad réside dans un Islam spirituel, fait de contemplation et de sagesse.

Une simple phrase prononcée par Saadi, le marin du navire "Ibn Batouta", "S'il n'y avait pas la religion, les gens seraient bien plus heureux. » (ÉNARD, 2012: 141), invite Lakhdar à tout remettre en cause.

Saadi est donc le porte-parole de Mathias Énard qui semble professer le déisme dans son roman. Selon lui, la religion sème la zizanie entre les gens et crée les germes de l'intolérance et de la violence entre les êtres. En analysant la scène ci-dessous, nous constaterons comment Lakhdar parvient à se débarrasser de tout ce qui le lie à la religion ou à l'extrémisme qui en découle:

"Je l'ai remercié (Cheikh Nouredine) sans oser lui parler de mon rêve, cette petite librairie religieuse et païenne à la fois dans le Raval à Barcelone. » (ÉNARD, 2012: 238)

À nos yeux, la petite librairie (dont rêve Lakhdar) constitue une sorte de "voyage immobile, une porte ouverte sur un ailleurs, quelque chose d'unique, un trésor »(COLINSIMARD, 1996: 68). Ce jeune homme constate alors que le vrai musulman doit accepter l'Autre, quelle que soit sa croyance. La preuve en est que le Coran rend hommage aux " Gens de la Caverne ", qui adoptaient la religion chrétienne, mais aussi à Alexandre le Grand (qui était païen):

"J'ai écouté le prône qui était retransmis par les hautparleurs, il était

question de la sourate des Gens de la Caverne et des voyages 
d'Alexandre au pays de Gog et Magog; l'Imam était savant et pieux, un homme sage peu versé dans la politique; (...) » (ÉNARD, 2012: 58)

C'est ainsi que Lakhdar connaît sa propre voie et commence à voir les êtres et le monde d'une manière tout à fait différente. La tolérance qu'il incarnait, dès le début du roman, lui a permis de créer un nouveau modèle culturel fondé sur l'ouverture sur l'Autre, même sur les peuples païens. La tolérance se double ainsi d'un déisme absolu qui l'invite à accepter les différentes religions et croyances.

Le héros de Rue des voleurs n'a donc rien à craindre. Si, au début de l'histoire, il a été impliqué dans le Mal malgré lui, la clôture du roman témoigne de la modification salutaire qui le touche au plan intellectuel et humain. Qu'importe donc s'il tombe dans les mains des policiers ou des juges? Qu'importe si on l'emprisonne ou si on l'exécute? En fait, ce qui compte, à nos yeux, c'est le message de paix et de tolérance qu'il lègue au lecteur à travers son comportement innocent et indulgent. Ce qui compte, c'est qu'il n'a jamais été une marionnette, un « Je servile » (KRISTEVA, 1977: 116) dans les mains des Islamistes et qu'il était un "Je souverain » (KRISTEVA, 1977: 116) qui réfléchit, qui lutte contre le dogmatisme et qui applique sa raison. Bref, il avait un esprit éclairé.

\section{Conclusion.}

Dans Rue de voleurs, nous avons égard à une " hétérogénéité du

matériel narratif »(BONI, 2011: 46). En fait, Mathias Énard mêle les genres et les styles: récit; discours biblique; discours coranique qui, à son tour, mêle le style épique aux tendances spirituelles; poésie; littératures arabe, francophone et espagnole.

Pour ce qui est du mythe du péché originel, il nourrit le roman d'Énard où nous avons égard à une malédiction initiale 
ayant permis à Lakhdar de faire peau neuve. En fait, si le péché originel prive le héros des bienfaits dont il jouissait autrefois, l'expulsion du paradis terrestre contribue à son apprentissage et à sa formation idéologique.

À chaque volet de cette recherche, nous avons souligné les contrastes qui servent à traduire les errances de Lakhdar ainsi que les contradictions et les mystères du monde. La double image de Tanger (décrite dans le roman d'Ángel Vázquez) renvoie au double aspect de la vie humaine: contrainte et liberté / souffrance et plaisir. Même le Coran fait allusion à la double face de la religion: Islam politique jetant la lumière sur le rôle de la bataille destinée à défendre la religion, d'une part, et Islam ascétique faisant prévaloir le rôle du recueillement et de la contemplation, d'autre part.

Si Énard insère des vers écrits en arabe dans son roman, c'est pour nous montrer que les Arabes ne sont pas tout à fait lubriques ou qu'ils sont uniquement attirés par l'amour charnel et prosaïque. Au contraire, le poète arabe est parvenu à doter la femme d'une valeur idéale et à la sortir de ce moule humiliant qui fait d'elle une femme objet. Par ailleurs, la poésie de Kabbani montre que le héros est tiraillé entre innovation et imitation servile. Cependant, il finit par plagier les vers des Autres car le patrimoine culturel et littéraire ressemble bien au paradis perdu. Tous les deux n'ont pas de substituts. Tout ce qui est ancien, dispose ainsi d'un charme irrésistible.

À nos yeux, l'intertexte n'a pas de valeur statique. C'est là que réside le secret de la créativité littéraire; bien qu'il puise dans les écritures antérieures, l'auteur de Rue des voleurs est apte à l'innovation. Avec Énard, l'intertextualité n'est pas «un jeu d'énigmes » (WACH, 2012: 365) puisqu'il cite directement la source, la référence de l'intertexte et qu'il ne fait pas, en conséquence, appel à la compétence culturelle du lecteur. Pourtant, il fait toujours appel à la compétence intellectuelle $\mathrm{du}$ récepteur car la valeur de l'intertexte diffère selon le 
contexte du roman. Ainsi la méthode comparative que nous avons employée met-elle l'accent sur l'originalité de Rue des voleurs. En fait, ce roman ne se contente pas de rassembler les textes antérieurs, mais il les dote également d'une valeur supplémentaire. Chez Énard, l'intertextualité est toujours «la recherche d'un effet de sens »(WACH, 2012: 365).

\section{BIBLIOGRPAHIE}

\section{- Le Corpus:}

ÉNARD, Mathias (2012). Rue des voleurs. France. Actes sud. Leméac éditeur.

I) Ouvrages consacrés à la littérature comparée:

- CLAUDON, Francis \& al. (1992). Précis de littérature comparée. Théories et méthodes de l'approche comparatiste. Paris. Nathan.

- DETHURENS, Pascal \& al. (2000). Fin d'un Millénaire: Rayonnement de la Littérature Comparée. France. Presses Universitaires de Strasbourg.

- PAGEAUX, Danie-Henri (1994). La Littérature générale et comparée. Paris. Armand Colin Éditeur.

- SOUILLER, Didier \& al. ( 1997). Littérature comparée. Paris. Presses Universitaires de France.

\section{II) Ouvrages consacrés à la critique littéraire:}

- GENETTE, Gérard (1982). Palimpsestes. La littérature au second degré. Paris. Seuil.

- JAUSS, Hans Robert (1988). Pour une herméneutique littéraire. (Traduit de l'allemand par Maurice Jacob). France. Gallimard.

- KRISTEVA, Julia

*(1969). Sèméiotikè. Recherches pour une sémanalyse. Paris. Seuil.

* (1977). Polylogue. Paris. Seuil. 
-MAURON, Charles (1996). Des Métaphores Obsédantes Au Mythe Personnel. Introduction à la psychocritique. Tunis. Cérès Éditions.

- PIÉGAY-GROS, Nathalie (1996). Introduction à l'Intertextualité. Paris. Dunod.

- SAMOYAULT, Tiphaine (2005). L'Intertextualité, Mémoire de la littérature. Paris. Armand Colin Éditeur.

III) Ouvrages généraux:

1- Ouvrage consacré à la linguistique:

- KROH, Aleksandra (2000). L'aventure du bilinguisme. Paris. L'Harmattan.

\section{2- Ouvrages portant sur la religion:}

- La Bible- L'Ancien et le Nouveau Testament (1988). Paris. Le Cerf.

- La Traduction du Noble Coran en langue française (1424 de l'hégire). Al-Madinah Al-Munnawwarah. Royaume d'Arabie Saoudite. Le Complexe Roi Fahd pour l'impression du Noble Coran.

\section{3-Dictionnaires:}

- JULLIEN, Claudia (2003). Dictionnaire de la Bible dans la littérature française. Paris. Vuibert.

- MOEZZI, Mohammad Ali Amir (2007). Dictionnaire du Coran. Paris. Robert Laffont.

\section{4- Articles de périodiques:}

- COLIN-SIMARD, Valérie. «Freud ou la révélation du souci ». In Le Magazine Littéraire. N³45. Juillet 1996. 41-45.

- FAUCONNIER, Bernard. "L'âge classique: Le spectacle des passions ». In Le Magazine Littéraire. $\quad$ N$^{\circ} 455$. Juillet 2006. 47-49.

- MONTEAUX, Michel. «Le désir, ou la trahison du bonheur moderne ». In Le Magazine Littéraire. N455. Juillet 2006. 3033.

- RIFATERRE, Michael. « La trace de l'intertexte ». In La Pensée. N²15. Octobre 1980. 04-10. 


\section{IV) Webographie:}

\section{Thèses de doctorat:}

- BONI, Martha (2011) . De l'intertextualité au transmédial: Pratiques de réécriture autour de Romanzo Criminale. Université Sorbonne Nouvelle. France. (Consultée le 16 janvier 2019). http: //www.theses.fr.

- WACH, Aurélie (2012). L'intertextualité comme procédé dramaturgique dans Hécube et les Troyennes d'Euripide.

Université Lille 3- Charles de Gaulle. France. (Consultée le 17 janvier 2020). http: // eprints_Phd.biblio.unitn.it. 